\title{
Reply to Letter to the Editor regarding our Paper: How to Intervene in the Caries Process: Early Childhood Caries - A Systematic Review
}

\author{
Julian Schmoeckel ${ }^{a} \quad$ Kristina Gorseta $^{b} \quad$ Christian H. Splieth $^{a}$ Hrvoje Juric $^{b}$ \\ ${ }^{a}$ Department of Preventive and Paediatric Dentistry, University of Greifswald, Greifswald, Germany; ${ }^{b}$ Department of \\ Paediatric and Preventive Dentistry, School of Dental Medicine, University of Zagreb, Zagreb, Croatia
}

Dear Editor,

We highly appreciate that a team of researchers intensively read our article and was willing to invest time and effort to comment on the publication. The comments are helpful, but they do not change the content of the publication. The paper was critically revised in the reviewing process and the key messages confirmed by the opinions of 24 experts in "How to Intervene in the Caries Process in Children: A Joint ORCA and EFCD Expert Delphi Consensus Statement" [Splieth et al., 2020].

Regarding the type of article (systematic review), like the Cochrane Foundation, we faced the problem that the available publications were not designed primarily to make systematic reviews feasible. Thus, systematic reviews often state that no high-level evidence-based conclusions can be drawn, which does not really suffice if you have to reach decisions in clinics or draw conclusions in an expert panel.

Thus, the design of the paper, i.e., starting with a systematic review, was due to the work being part of a requested review consortium for a consensus paper of the European Organisation for Caries Research and the European Federation of Conservative Dentistry regarding caries management. The aim was to provide the most re- cent literature in the field to be able to provide the expert panel with a beneficial, clinically relevant, scientific background. The search strategy was systematic, but due to the nature of the available literature, studies other than randomized controlled trials (RCTs) were included. In these studies, deviations from a "pure" systematic review for RCTs only were necessary and can be traced in the paper. After screening and reading the retrieved papers, we found different levels of evidence regarding different methods for the management of early childhood caries (e.g., several systematic reviews regarding nonoperative caries management $[\mathrm{NOCM}]$ with silver diamine fluoride [SDF]). We thus divided the topics into 3 different subaspects (NOCM with SDF, NOCM other than SDF, and restorative), adapting the evaluation and presenting the data as pragmatically and clearly as possible.

It is correct that there are many tools for assessment of the primary literature for a systematic review, e.g., the PRISMA checklist and the Newcastle-Ottawa Scale (NOS), which we used. We appreciate the recommendation of further assessment tools as mentioned in Ma et al. [2020]. Nonetheless, retrospective rating of primary references will always include a subjective evaluation process, given the nature of heterogenic reporting. Regarding 
the assessment in the NOCM group containing randomized controlled trials (RCTs) and cohort studies, a comparable analysis for the risk of bias and quality assessment was complicated due to the extremely heterogenic character of the underlying research publications.

Wells et al. [2016] was cited correctly in the text but was missing in the list of references. We apologize for this omission.

Regarding one of the cohort studies on the NOCM of ECC, Peretz and Gluck [2006], it is correct that proximal slicing was performed and that this is a nonrestorative/ nonoperative cavity treatment. Still, within the abovementioned division of the topics into the 3 different subaspects (NOCM with SDF, NOCM other than SDF, and restorative), this study clearly belongs to NOCM. Naming this group more specifically "nonrestorative caries management" (NRCM) would exclude any doubts. However, this is actually the smallest part of the problem when assessing NOCM or NRCM. The treatments reported vary a lot, as do the cohorts where they were performed, and the adequate control group was also often missing.

Regarding the PRISMA flow diagram, unfortunately these miscalculations/typing errors were not eradicated in the review process.

Regarding the following sentence "SDF solution applied once or twice a year could arrest active caries lesions involving dentine with a success rate of 79 and $91 \%$, respectively," we could, as suggested, rather have cited Zhi et al. [2012] or Duangthip et al. [2015]. With respect to retrieving articles from other sources, it is clear that (systematic) reviews will automatically encounter references from previous reviews. This actually proves the quality of the search strategy.

Regarding the comment on the inclusion of Gao et al. [2016], it is correct that their paper did not only contain studies on primary teeth. We discussed this with the research team and decided to still include this paper, as the relevant content regarding primary teeth can be retrieved independently and it addresses a clinically relevant aspect. Excluding this study would have had no relevant impact on the key messages of the review.

We understand that a different research team might have made different choices at certain stages of the reviewing process, especially when assessing bias and determining the dichotomized decision of inclusion or exclusion. Still, we think we tackled the frequent problem of pure systematic reviews often stating that "there is not enough evidence/not enough studies to meet inclusion criteria" and the final outcome or conclusion in these cases is that "more studies with that specific question are needed." With our open approach, we could lay the best available ground for the expert consensus by ORCA and EFCD.

We hope our answers are helpful and clarify the concerns expressed. We are grateful for the response as we are always willing to improve our further scientific work.

\section{References}

Duangthip D, Jiang M, Chu CH, Lo EC. Non-surgical treatment of dentin caries in preschool children - systematic review. BMC Oral Health. 2015 Apr;15(1):44.

Gao SS, Zhang S, Mei ML, Lo EC, Chu CH. Caries remineralisation and arresting effect in children by professionally applied fluoride treatment - a systematic review. BMC Oral Health. 2016 Feb;16(1):12.

Ma LL, Wang YY, Yang ZH, Huang D, Weng H, Zeng XT. Methodological quality (risk of bias) assessment tools for primary and sec- ondary medical studies: what are they and which is better? Mil Med Res. 2020 Feb;7(1): 7.

Peretz B, Gluck G. Early childhood caries (ECC): a preventive-conservative treatment mode during a 12-month period. J Clin Pediatr Dent. 2006;30(3):191-4.

Splieth CH, Banerjee A, Bottenberg P, Breschi L, Campus G, Ekstrand KR, et al. How to Intervene in the Caries Process in Children: A Joint ORCA and EFCD Expert Delphi Consensus Statement. Caries Res. 2020;54(4):297-305.
Wells G, Shea B, O'Connell D, Robertson J, Peterson J, Welch V, et al. [Internet] The Newcastle-Ottawa Scale (NOS) for assessing the quality of nonrandomized studies in meta-analysis [cited 2016]. Available from: http://www. evidencebasedpublichealth.de/download/ Newcastle_Ottowa_Scale_Pope_Bruce.pdf

Zhi QH, Lo EC, Lin HC. Randomized clinical trial on effectiveness of silver diamine fluoride and glass ionomer in arresting dentine caries in preschool children. J Dent. 2012 Nov; 40(11):962-7. 